\title{
Analysis of a Time Implicit Scheme for the Oseen Model Driven by Nonlinear Slip Boundary Conditions
}

\author{
J. K. Djoko and J. M. Lubuma
}

Department of Mathematics and Applied Mathematics, University of Pretoria, Pretoria, South Africa, e-mail: jules.djokokamdem@up.ac.za ; e-mail: jean.lubuma@up.ac.za

\begin{abstract}
This work is concerned with the time discrete analysis of the Oseen system of equations driven by nonlinear slip boundary conditions of friction type. We study the existence of solutions of the time discrete model and derive several a priori estimates needed to recover the solution of the continuous problem by means of weak compactness. Moreover, for the difference between the exact and approximate solutions, we obtain the rate of convergence of order one with respect to the time step without imposing extra regularity on the weak solution.
\end{abstract}

Mathematics Subject Classification. 65M12, 76D05.

Keywords. Oseen equations, nonlinear slip boundary conditions, variational inequality, time discrete approximation, convergence, regularity of solutions, rate of convergence.

\section{Introduction}

The objective of this work is to study the convergence without regularity assumptions on the exact solutions of the time discrete two dimensional Oseen equations driven by nonlinear slip boundary conditions of friction type. Qualitative analysis of the time discrete model such as 2d Navier Stokes equations with Dirichlet boundary conditions, has been studied in [1-4]. In [1], several time discrete model of Navier Stokes are formulated and their stability analysis presented with great details. Ju [2] has formulated a linearized approximation of the Navier Stokes equations and derived several a priori bound for the discrete solution. In fact $H^{2}$ uniform a priori bound is obtained with the aid of several versions of Gronwall's Lemmas. In [3], Tone and Wirosoetisno have considered the fully implicit Euler time approximation and proved $H^{1}$-uniform stability (see also $[4,5]$ where several extensions, and simplifications of initial proofs are presented).

Based on the work of Temam (see [1]), it is clear that existence theories for evolution problem can be obtained by first time discretization, derivation of a priori estimates uniform with respect to the discretization parameter, and lastly application of weak convergence results. A key step in that simple description is then to prove convergence of the semi-discrete approximations to solutions of the continuous/original formulation. If moreover, one wants to derive error estimates, then the question of rate of convergence versus the regularity of the exact solution is of major interest in the development of efficient/reliable numerical schemes. It is the the objective of this study to show that if one adopts implicit time approximations for the Oseen model governed by nonlinear slip boundary conditions of friction type, optimal rate of convergence with respect to the discretization parameter can be obtained by maintaining the regularity of the exact solution but under moderate regularity on the forcing term. Our work is inspired by seminal contribution of Nochetto et al. [6] (see also [7]).

$\mathrm{Li}$ and $\mathrm{Li}$ in [8] have studied the 2d Navier Stokes equations and derived optimal convergence provided that the exact solution is twice differentiable in time. The method of proof in [8] make use of the Taylor's expansion, hence more regularity is needed. In this work instead we will follow the approach advocate in $[6,7]$ which control the consistency error by introducing discrete dissipative (non negative) quantities 
and careful utilization of sub-differential operator. To the best of our knowledge, almost all works in the literature for fluids flow driven by nonlinear slip boundary conditions of friction type are concerned with existence of solutions or its finite element discretizations (see [8-17] just to mention a few). A fully discrete approximation of the Navier Stokes system with Tresca boundary condition has been considered in $\mathrm{Li}$ and $\mathrm{Li}$ in [8], but in order to obtain convergence for the discretization in time higher regularity of the exact solution is needed. We regard our convergence analysis with minimal regularity on the weak solution as important for problem whose time discrete model are formulated as (2.20). Similar study has been contributed recently by Bartels (see [18]) for a class of rate independent evolution problems, and we believe this new approach for convergence analysis will gain more popularity in the community.

The rest of the work is organized as follows. In Sect. 2, we introduce the equations and formulate the variational inequality associated to the problem, we fix the notations and introduce the time discrete implicit scheme associated to the continuous problem. Section 3 deals with the derivation of some a priori estimates which are later exploited to obtain the solution of the continuous problem. Moreover, we also show the convergence of the discrete solution by means of some weak and strong convergence results. Section 4 is about the convergence with minimal regularity assumptions on the exact solution.

\section{Preliminaries}

\subsection{Model Problem: Variational Formulation}

Let $\Omega \subset \mathbb{R}^{2}$ be an open bounded set with boundary $\partial \Omega$ assume to be regular enough, and fix $T>0$. We are interested in the time approximation of the incompressible Oseen system of equations

$$
\left\{\begin{array}{l}
\boldsymbol{u}_{t}-\nu \Delta \boldsymbol{u}+(\boldsymbol{w} \cdot \nabla) \boldsymbol{u}+\nabla p=\boldsymbol{f} \quad \text { in } \Omega \times(0, T), \\
\operatorname{div} \boldsymbol{u}=0 \quad \text { in } \Omega \times(0, T)
\end{array}\right.
$$

where $\boldsymbol{u}=\left(u_{1}, u_{2}\right)$ is the velocity, the pressure is $p(\boldsymbol{x}, t)$ and $\boldsymbol{f}(\boldsymbol{x}, t)$ is the external body force applied to the fluids. $\nu$ is the kinematic viscosity. $\boldsymbol{w}$ is a known quantity that satisfies

$$
\operatorname{div} \boldsymbol{w}=0 \quad \text { on } \Omega \times(0, T) .
$$

Further regularity on $\boldsymbol{w}$ will be given later, and we assume that $\boldsymbol{w}$ is independent of time. The equations in (2.1) are supplemented by the initial condition

$$
\boldsymbol{u}(\boldsymbol{x}, 0)=\boldsymbol{u}_{0} \text { on } \bar{\Omega},
$$

with $\boldsymbol{u}_{0}: \bar{\Omega} \longmapsto \mathbb{R}^{2}$ given, and precise assumptions will be introduced below, and $\bar{\Omega}$ is the closure of $\Omega$. Next in order to describe the motion of the fluid on the the boundary $\partial \Omega$, we first assume that $\partial \Omega$ is made of two components $S$ and $\Gamma$, such that $\partial \Omega=\bar{S} \cup \bar{\Gamma}$, with $S \cap \Gamma=\emptyset$. We assume the homogeneous Dirichlet condition on $\Gamma$, that is

On $S$, we first assume the impermeability condition

$$
\boldsymbol{u}=\mathbf{0} \quad \text { on } \Gamma \times(0, T) .
$$

$$
u_{N}=\boldsymbol{u} \cdot \boldsymbol{n}=0 \quad \text { on } S \times(0, T),
$$

where $\boldsymbol{n}$ is the outward unit normal on the boundary $\partial \Omega$, and $u_{N}$ is the normal component of the velocity, while $\boldsymbol{u}_{\boldsymbol{\tau}}=\boldsymbol{u}-u_{N} \boldsymbol{n}$ is its tangential component. In addition to (2.4) we also impose on $S$, a threshold slip condition $[10,11]$, which can be formulated as follows; given a non negative function $g: S \longrightarrow(0, \infty)$, one has the following

$$
\left.\begin{array}{l}
\text { if } \quad\left|(\boldsymbol{T n})_{\boldsymbol{\tau}}\right|<g \quad \text { then } \quad \boldsymbol{u}_{\boldsymbol{\tau}}=\mathbf{0}, \\
\text { if } \quad\left|(\boldsymbol{T} \boldsymbol{n})_{\boldsymbol{\tau}}\right|=g \text { then } \quad \boldsymbol{u}_{\boldsymbol{\tau}} \neq \mathbf{0}, \quad \text { and }-(\boldsymbol{T} \boldsymbol{n})_{\boldsymbol{\tau}}=g \frac{\boldsymbol{u}_{\boldsymbol{\tau}}}{\left|\boldsymbol{u}_{\boldsymbol{\tau}}\right|}
\end{array}\right\} \quad \text { on } S \times(0, T),
$$

where $|\boldsymbol{v}|^{2}=\boldsymbol{v} \cdot \boldsymbol{v}$ is the Euclidean norm. Equation (2.5) expresses the fact that $(\boldsymbol{T n})_{\boldsymbol{\tau}}$ and $\boldsymbol{u}_{\boldsymbol{\tau}}$ are parallel but opposite. Of course in $(2.5), \boldsymbol{T}=2 \nu \varepsilon(\boldsymbol{u})-p \boldsymbol{I}$ is the Cauchy stress tensor with $\boldsymbol{I}$ being 
the identity tensor and $\varepsilon(\boldsymbol{u})=\frac{1}{2}\left(\nabla \boldsymbol{u}+(\nabla \boldsymbol{u})^{T}\right)$. For the mathematical setting of our work, we shall follow Temam [1] and introduce standard definitions and facts similar for the treatment of Navier Stokes equations in general, but because of (2.5), some minor modifications will be made clear when necessary. Throughout the paper, $C$ denotes a generic positive constants which may take different values even in the same calculation. The entities on which may depend, are given in brackets, e.g., $C(\Omega)$ denotes a constant which depends at most on $\Omega$. As usual, $\phi(t)$ stands for the function $\boldsymbol{x} \in \Omega \mapsto \phi(\boldsymbol{x}, t)$. Standard notation on Lebesgue and Sobolev spaces is employed and $(\cdot, \cdot)$ denotes the $L^{2}$ scalar product, and $\|\cdot\|$ the $L^{2}$-norm.

Let $\Psi: \boldsymbol{X} \rightarrow \mathbb{R}$, with $\boldsymbol{X}$ being a Hilbert space, and $\boldsymbol{X}^{\prime}$ its dual. We denote by $\langle\cdot, \cdot\rangle$ the duality pairing between $\boldsymbol{X}$ and $\boldsymbol{X}^{\prime}$. Let $x_{0} \in \boldsymbol{X}$, then

$$
y \in \partial \Psi\left(x_{0}\right) \text { if and only if } \Psi(x)-\Psi\left(x_{0}\right) \geq\left\langle y, x-x_{0}\right\rangle, \quad \forall x \in \boldsymbol{X} .
$$

With (2.6) in mind, (2.5) is re-written as follows (see [19]):

$$
-(\boldsymbol{T n})_{\boldsymbol{\tau}} \in g \partial\left|\boldsymbol{u}_{\boldsymbol{\tau}}\right| \text { on } S \times(0, T),
$$

where $\partial|\cdot|$ is the sub-differential of the real valued function $|\cdot|$.

Throughout this work, boldface characters denote vector quantities, and $\boldsymbol{H}^{1}(\Omega)=H^{1}(\Omega)^{2}$ and $\boldsymbol{L}^{2}(\Omega)=L^{2}(\Omega)^{2}$. For a mathematical formulation of the problem, we introduce the following functions spaces and functionals [1].

$$
\begin{aligned}
\mathbb{V} & =\left\{\boldsymbol{v} \in \boldsymbol{H}^{1}(\Omega):\left.\boldsymbol{v}\right|_{\Gamma}=0,\left.\boldsymbol{v}_{n}\right|_{S}=0\right\}, \\
\mathbb{V}_{\mathrm{div}} & =\left\{\boldsymbol{u} \in \boldsymbol{H}^{1}(\Omega), \operatorname{div} \boldsymbol{u}=0,\left.\boldsymbol{u}\right|_{\Gamma}=\mathbf{0},\left.\boldsymbol{u} \cdot \boldsymbol{n}\right|_{S}=0\right\}, \\
\mathbb{H} & =\left\{\boldsymbol{u} \in \boldsymbol{L}^{2}(\Omega), \operatorname{div} \boldsymbol{u}=0,\left.\boldsymbol{u} \cdot \boldsymbol{n}\right|_{\partial \Omega}=0\right\} .
\end{aligned}
$$

We introduce the continuous bilinear form $a(\cdot, \cdot)$ given as follows

$$
\begin{aligned}
a: \mathbb{V} \times \mathbb{V} & \longmapsto \mathbb{R} \\
\quad(\boldsymbol{v}, \boldsymbol{u}) & \longrightarrow a(\boldsymbol{v}, \boldsymbol{u})=2 \nu(\varepsilon(\boldsymbol{v}), \varepsilon(\boldsymbol{u})) .
\end{aligned}
$$

At this point we recall that the Korn inequality reads: there is a constant $C_{K}$ depending only on $\Omega$ such that

$$
C_{K} \int_{\Omega} \nabla \boldsymbol{v}: \nabla \boldsymbol{v} d x \leq \int_{\Omega} \varepsilon(\boldsymbol{v}): \varepsilon(\boldsymbol{v}) d x \quad \text { for all } \boldsymbol{v} \in \mathbb{V}
$$

while the Poincaré-Friedrichs inequality state that; there is constant $C_{P}$ depending only on the domain $\Omega$ such that

$$
C_{P} \int_{\Omega}|\boldsymbol{v}|^{2} d x \leq \int_{\Omega} \nabla \boldsymbol{v}: \nabla \boldsymbol{v} d x \quad \text { for all } \boldsymbol{v} \in \mathbb{V} .
$$

Thus, it is manifest that the norms $\|\cdot\|_{1}$ and $\|\nabla \cdot\|$ are equivalent on $\mathbb{V}$. The norm on $\mathbb{V}$ will be denoted as $\|\cdot\|_{\mathbb{V}}$. Thus with $(2.8)$, one easily see that

$$
2 \nu C_{K}\|\boldsymbol{v}\|_{\mathbb{V}}^{2} \leq a(\boldsymbol{v}, \boldsymbol{v}) \text { for all } \boldsymbol{v} \in \mathbb{V} .
$$

From the continuity of $a(\cdot, \cdot)$, the Riesz representation theorem is applicable and there is a bounded operator $\mathcal{A}: \mathbb{V} \rightarrow \mathbb{V}^{\prime}$ such that $a(\boldsymbol{u}, \boldsymbol{v})=\langle\mathcal{A} \boldsymbol{u}, \boldsymbol{v}\rangle$.

Next, we introduce the second bilinear form $b_{\boldsymbol{w}}(\cdot, \cdot)$ given as follows:

$$
\begin{aligned}
b_{\boldsymbol{w}}: \mathbb{V} \times \mathbb{V} & \longmapsto \mathbb{R} \\
(\boldsymbol{u}, \boldsymbol{v}) & \longmapsto b_{\boldsymbol{w}}(\boldsymbol{u}, \boldsymbol{v})=((\boldsymbol{w} \cdot \nabla) \boldsymbol{u}, \boldsymbol{v}) .
\end{aligned}
$$

One can show that $b_{\boldsymbol{w}}(\cdot, \cdot)$ is continuous on $\boldsymbol{H}^{1}(\Omega)$, and enjoys the following properties for $(\boldsymbol{u}, \boldsymbol{v}) \in \mathbb{V}^{2}$ and $\boldsymbol{w} \in \mathbb{V}_{\text {div }}$

$$
\begin{aligned}
\left|b_{\boldsymbol{w}}(\boldsymbol{v}, \boldsymbol{u})\right| & \leq C\|\boldsymbol{w}\|^{1 / 2}\|\nabla \boldsymbol{w}\|^{1 / 2}\|\nabla \boldsymbol{u}\|\|\boldsymbol{u}\|^{1 / 2}\|\nabla \boldsymbol{u}\|^{1 / 2}, \\
b_{\boldsymbol{w}}(\boldsymbol{v}, \boldsymbol{v}) & =0,
\end{aligned}
$$

the last property implying

$$
b_{\boldsymbol{w}}(\boldsymbol{u}, \boldsymbol{v})=-b_{\boldsymbol{w}}(\boldsymbol{v}, \boldsymbol{u}) \text { for all } \boldsymbol{u}, \boldsymbol{v} \in \mathbb{V}
$$


From the Riesz representation theorem, there is a bounded operator $\mathcal{B}_{\boldsymbol{w}}: \mathbb{V} \rightarrow \mathbb{V}^{\prime}$ such that $b_{\boldsymbol{w}}(\boldsymbol{u}, \boldsymbol{v})=$ $\left\langle\mathcal{B}_{\boldsymbol{w}} \boldsymbol{u}, \boldsymbol{v}\right\rangle$. We assume that

$$
(\boldsymbol{f}, g) \in L^{\infty}\left(0, T ; \boldsymbol{L}^{2}(\Omega)\right) \times L^{2}(S) .
$$

Finally, we introduce the functionals (continuous on $\mathbb{V}$ )

$$
\begin{aligned}
J: \mathbb{V} & \longmapsto \mathbb{R} \\
\boldsymbol{v} & \longmapsto J(\boldsymbol{v})=\left(g,\left|\boldsymbol{v}_{\boldsymbol{\tau}}\right|\right)_{S}, \\
\ell: \mathbb{V} & \longmapsto \mathbb{R} \\
\boldsymbol{v} & \longmapsto \ell(\boldsymbol{v})=(\boldsymbol{f}, \boldsymbol{v}) .
\end{aligned}
$$

We want to analyze the discrete in time approximation of the solution of the following evolution problem: Find $\boldsymbol{u}(t) \in \mathbb{V}_{\text {div }}$, such that

$$
\left\{\begin{array}{l}
\boldsymbol{u}(0)=\boldsymbol{u}_{0} \text { in } \Omega \\
\text { and for all } \boldsymbol{v} \in \mathbb{V}_{\mathrm{div}}, \text { and a.e } t>0, \\
\left\langle\boldsymbol{u}^{\prime}(t), \boldsymbol{u}(t)-\boldsymbol{v}\right\rangle+a(\boldsymbol{u}(t), \boldsymbol{u}(t)-\boldsymbol{v})+b_{\boldsymbol{w}}(\boldsymbol{u}(t), \boldsymbol{u}(t)-\boldsymbol{v}) \\
+J(\boldsymbol{u}(t))-J(\boldsymbol{v}) \leq \ell(\boldsymbol{u}(t)-\boldsymbol{v})
\end{array}\right.
$$

Of course the pressure is recovered in the usual way following [13]. Kashiwabara in [16], has obtained the well posedness of the full Navier Stokes system associated to (2.15) by adopting a proof which rely on; regularization of the non-differentiable functional $J$, together with Faedo-Galerkin and compactness arguments. We point out that similar arguments can be employed here because (2.15) can be regarded as "linearized version" of Navier Stokes equations, in fact the existence of solutions of (2.15) will be a consequence of the analysis that will be presented in Sect. 3 .

Remark 2.1. The variational inequality (2.15) is equivalent to the evolutionary inclusion

$$
\left\{\begin{array}{l}
\text { find } \boldsymbol{u}(t) \in \mathbb{V}_{\text {div }}, \text { such that } \\
\boldsymbol{u}(0)=\boldsymbol{u}_{0} \text { in } \Omega \\
-\boldsymbol{u}^{\prime}(t)-\mathcal{A} \boldsymbol{u}(t)-\mathcal{B}_{\boldsymbol{w}} \boldsymbol{u}(t)+\boldsymbol{f}(t) \in \partial J(\boldsymbol{u}(t))
\end{array}\right.
$$

Since $J$ is l.s.c and convex, (2.16) is equivalent to

$$
\left\{\begin{array}{l}
\text { find } \boldsymbol{u}(t) \in \mathbb{V}_{\text {div }}, \text { such that } \\
\boldsymbol{u}(0)=\boldsymbol{u}_{0} \text { in } \Omega \\
\boldsymbol{u}(t) \in \partial J^{*}\left(-\boldsymbol{u}^{\prime}(t)-\mathcal{A} \boldsymbol{u}(t)-\mathcal{B}_{\boldsymbol{w}} \boldsymbol{u}(t)+\boldsymbol{f}(t)\right),
\end{array}\right.
$$

where $J^{*}$ is the Legendre transform of $J$. Having in mind that $J$ is degree one homogeneous, one gets

$$
J^{*}(\boldsymbol{w})=\boldsymbol{I}_{\partial J(0)}(\boldsymbol{w})=\left\{\begin{array}{l}
0, \boldsymbol{w} \in \partial J(0), \\
\infty, \boldsymbol{w} \notin \partial J(0) .
\end{array}\right.
$$

Thus (2.17) becomes (using (2.5) and (2.6))

$$
\left\{\begin{array}{l}
\text { find } \boldsymbol{u}(t) \in \mathbb{V}_{\text {div }}, \text { such that } \\
\boldsymbol{u}(0)=\boldsymbol{u}_{0} \text { in } \Omega \\
-\boldsymbol{u}^{\prime}(t)-\mathcal{A} \boldsymbol{u}(t)-\mathcal{B}_{\boldsymbol{w}} \boldsymbol{u}(t)+\boldsymbol{f}(t) \in \partial J(0), \\
\frac{1}{2} \frac{d}{d t}\|\boldsymbol{u}(t)\|^{2}+a(\boldsymbol{u}(t), \boldsymbol{u}(t))+\left\langle\boldsymbol{v}^{*}, \boldsymbol{u}(t)\right\rangle \leq \ell(\boldsymbol{u}(t)), \text { for all } \boldsymbol{v}^{*} \in \partial J(0) .
\end{array}\right.
$$

The equivalent variational problem (2.18) has not yet been used in the literature, and in the near future we intend to do so.

For the time discretization of (2.14), we divide the interval $(0, T)$ into $N$ sub intervals of equal size $k=t_{n}-t_{n-1}=T / N$. we shall introduce by induction a sequence of elements of $\mathbb{V}$, say $\boldsymbol{u}_{0}^{k}, \boldsymbol{u}_{1}^{k}, \ldots, \boldsymbol{u}_{N}^{k}$, 
where $\boldsymbol{u}_{n}^{k}$ is an approximation of $\boldsymbol{u}(t)$ we are seeking on the interval $I_{k, n}=\left(t_{n-1}, t_{n}\right)$ with $t_{n}=n k$. We approximate $\boldsymbol{f}$ by $\boldsymbol{f}_{n}^{k}$ given as follows

$$
\boldsymbol{f}_{n}^{k}=\frac{1}{k} \int_{t_{n-1}}^{t_{n}} \boldsymbol{f}(t) d t, \text { for } n=1,2,3, \ldots N, \quad \text { and } \boldsymbol{f}_{0}^{k}=\boldsymbol{f} .
$$

The discrete derivative in time is

$$
\delta \boldsymbol{u}_{n}^{k}=\frac{\boldsymbol{u}_{n}^{k}-\boldsymbol{u}_{n-1}^{k}}{k} \text { for } n=1,2,3, \ldots, N .
$$

We consider a time discretization of (2.19) using the implicit Euler scheme

$$
\left\{\begin{array}{l}
\text { Let } \boldsymbol{u}_{0}^{k}=\boldsymbol{u}_{0}, \\
\text { Find } \boldsymbol{u}_{n}^{k} \in \mathbb{V}_{\text {div }}, \text { such that for all } \boldsymbol{v} \in \mathbb{V}_{\text {div }}, \text { and } n=1,2, \ldots, N, \\
\left\langle\delta \boldsymbol{u}_{n}^{k}, \boldsymbol{u}_{n}^{k}-\boldsymbol{v}\right\rangle+a\left(\boldsymbol{u}_{n}^{k}, \boldsymbol{u}_{n}^{k}-\boldsymbol{v}\right)+b_{\boldsymbol{w}}\left(\boldsymbol{u}_{n}^{k}, \boldsymbol{u}_{n}^{k}-\boldsymbol{v}\right) \\
+J\left(\boldsymbol{u}_{n}^{k}\right)-J(\boldsymbol{v}) \leq\left(\boldsymbol{f}_{n}^{k}, \boldsymbol{u}_{n}^{k}-\boldsymbol{v}\right) .
\end{array}\right.
$$

Remark 2.2. If the field $\boldsymbol{w}$ depend on time, then we replace $b_{\boldsymbol{w}}(\cdot, \cdot)$ by $b_{\boldsymbol{w}_{n}^{k}}(\cdot, \cdot)$ where $\boldsymbol{w}_{n}^{k} \in \mathbb{V}_{\text {div }}$ is a time approximation of $\boldsymbol{w}$ with the property that $\boldsymbol{w}_{n}^{k}$ tends to $\boldsymbol{w}$ in $\boldsymbol{H}^{1}$ as the time step $k \rightarrow 0$.

With the sequence of approximate problems (2.20), the solvability of (2.14) will follow by adopting the method of proof advocate by Temam in [1] (Chap 3, pp. 216). So, we first show that (2.20) is well defined, next we pass to the limit as $k$ approaches zero after obtaining some uniform a priori estimates. Next, we derive a priori error estimates depending on the regularity of the datum. Our results can be interpreted in two different ways: (a) establishing existence and uniqueness of (2.14) via time discretization. (b) Prove numerical results such as convergence, stability of numerical solutions.

Computing the rate of convergence of the time discrete solution without extra regularity assumption on the weak solution is in our knowledge the first for fluid flow governed by nonlinear slip boundary conditions of friction type.

\subsection{Existence of (2.20)}

In this section we prove existence of solutions of (2.20). We argue inductively with respect to $n$, this is to say that we assume that we know $\boldsymbol{u}_{k}^{i}$ for $i=1,2, \ldots, n-1$ and we show that $\boldsymbol{u}_{k}^{n}$ is well defined via $(2.20)$. For that purpose, we first consider the case when $n=0$, because correspond a disturbance arising from (2.20). We indicate how the pressure is constructed if we know the velocity. To construct the pressure, it is important to recall the following result similar to the one in Glowinski [20] (Theorem 5.3, pp. 70).

Lemma 2.1. The variational problem (2.20) is equivalent to the following problem: find $\left(\boldsymbol{u}_{n}^{k}, \boldsymbol{\alpha}_{n}^{k}\right) \in \mathbb{V}_{\operatorname{div}} \times \boldsymbol{\Lambda}$ such that

$$
\left\{\begin{array}{l}
\text { Let } \boldsymbol{u}_{0}^{k}=\boldsymbol{u}_{0}, \\
\left\langle\delta \boldsymbol{u}_{n}^{k}, \boldsymbol{v}\right\rangle+a\left(\boldsymbol{u}_{n}^{k}, \boldsymbol{v}\right)+b_{\boldsymbol{w}}\left(\boldsymbol{u}_{n}^{k}, \boldsymbol{v}\right)+\left(g \boldsymbol{\alpha}_{n}^{k}, \boldsymbol{v}_{\boldsymbol{\tau}}\right)_{S}=\left(\boldsymbol{f}_{n}^{k}, \boldsymbol{v}\right), \\
\boldsymbol{\alpha}_{n}^{k} \cdot \boldsymbol{u}_{\boldsymbol{\tau}, n}^{k}=\left|\boldsymbol{u}_{\boldsymbol{\tau}, n}^{k}\right| \text { a.e on } S,
\end{array}\right.
$$

with

$$
\boldsymbol{\Lambda}=\left\{\boldsymbol{\alpha} \in \boldsymbol{L}^{\infty}(S) ;|\boldsymbol{\alpha}| \leq 1 \text { a.e. on } S\right\} .
$$

We introduce the linear mapping

$$
\mathcal{H}_{n}(\boldsymbol{v})=\left(\boldsymbol{f}_{n}^{k}, \boldsymbol{v}\right)-\left\langle\delta \boldsymbol{u}_{n}^{k}, \boldsymbol{v}\right\rangle-a\left(\boldsymbol{u}_{n}^{k}, \boldsymbol{v}\right)-b_{\boldsymbol{w}}\left(\boldsymbol{u}_{n}^{k}, \boldsymbol{v}\right)-\left(g \boldsymbol{\alpha}_{n}^{k}, \boldsymbol{v}_{\boldsymbol{\tau}}\right)_{S} .
$$

Clearly the mapping $\boldsymbol{v} \longrightarrow \mathcal{H}_{n}(\cdot)$ is continuous functional on $\boldsymbol{H}^{1}(\Omega)$, that vanishes on $\mathbb{V}_{\text {div }}$ if $\left(\boldsymbol{u}_{n}^{k}, \boldsymbol{\alpha}_{n}^{k}\right)$ are solution of (2.21). Thus from [21], for each interval $\left[t_{n-1}, t_{n}\right]$, there is an element $p_{n}^{k} \in L^{2}(\Omega)$ such that 


$$
\text { for all } \boldsymbol{v} \in \mathbb{V}, \int_{\Omega} p_{n}^{k} \operatorname{div} \boldsymbol{v} d x=\mathcal{H}_{n}(\boldsymbol{v}) \text {. }
$$

Since $\mathcal{H}_{n}(\cdot)$ vanishes on $\mathbb{V}_{\text {div }}$, we obtain that $\left(\operatorname{div} \boldsymbol{v}, p_{n}^{k}\right)=0$ for all $\boldsymbol{v} \in \mathbb{V}_{\text {div }}$. Which implies that $\left(p_{n}^{k}, 1\right)=0$, hence $p_{n}^{k} \in L_{0}^{2}(\Omega)$. Hence we conclude by saying that $\left(\boldsymbol{u}_{n}^{k}, \boldsymbol{\alpha}_{n}^{k}\right)$ is a solution of (2.21) means that $\left(\boldsymbol{u}_{n}^{k}, \boldsymbol{\alpha}_{n}^{k}, p_{n}^{k}\right)$ is a solution of

$$
\left\{\begin{array}{l}
\text { Let } \boldsymbol{u}_{0}^{k}=\boldsymbol{u}_{0}, \\
\text { for all }(\boldsymbol{v}, q) \in \mathbb{V} \times L_{0}^{2}(\Omega), \text { and } n=1,2,3, \ldots, N, \\
\left\langle\delta \boldsymbol{u}_{n}^{k}, \boldsymbol{v}\right\rangle+a\left(\boldsymbol{u}_{n}^{k}, \boldsymbol{v}\right)+b_{\boldsymbol{w}}\left(\boldsymbol{u}_{n}^{k}, \boldsymbol{v}\right)-\left(p_{n}^{k}, \operatorname{div} \boldsymbol{v}\right)+\left(g \boldsymbol{\alpha}_{n}^{k}, \boldsymbol{v}_{\boldsymbol{\tau}}\right)_{S}=\left(\boldsymbol{f}_{n}^{k}, \boldsymbol{v}\right), \\
\left(q, \operatorname{div} \boldsymbol{u}_{n}^{k}\right)=0 \\
\boldsymbol{\alpha}_{n}^{k} \cdot \boldsymbol{u}_{\boldsymbol{\tau}, n}^{k}=\left|\boldsymbol{u}_{\boldsymbol{\tau}, n}^{k}\right| \text { a.e on } S
\end{array}\right.
$$

which is also equivalent to (see Glowinski [20], Theorem 5.3, pp. 70)

$$
\left\{\begin{array}{l}
\text { Find }\left(\boldsymbol{u}_{n}^{k}, p_{n}^{k}\right) \in \mathbb{V} \times L_{0}^{2}(\Omega) \text { such that } \\
\boldsymbol{u}_{0}^{k}=\boldsymbol{u}_{0} \\
\text { for all }(\boldsymbol{v}, q) \in \mathbb{V} \times L_{0}^{2}(\Omega), \text { and } n=1,2, \ldots, N \\
\left\langle\delta \boldsymbol{u}_{n}^{k}, \boldsymbol{u}_{n}^{k}-\boldsymbol{v}\right\rangle+a\left(\boldsymbol{u}_{n}^{k}, \boldsymbol{u}_{n}^{k}-\boldsymbol{v}\right)+b_{\boldsymbol{w}}\left(\boldsymbol{u}_{n}^{k}, \boldsymbol{u}_{n}^{k}-\boldsymbol{v}\right)-\left(p_{n}^{k}, \operatorname{div}\left(\boldsymbol{u}_{n}^{k}-\boldsymbol{v}\right)\right) \\
+J\left(\boldsymbol{u}_{n}^{k}\right)-J(\boldsymbol{v}) \leq\left(\boldsymbol{f}_{n}^{k}, \boldsymbol{u}_{n}^{k}-\boldsymbol{v}\right) \\
\left(q, \operatorname{div} \boldsymbol{u}_{n}^{k}\right)=0
\end{array}\right.
$$

Next, the existence of the of velocity is obtained by first reformulating (2.20) as follow

$$
\left\{\begin{array}{l}
\text { Let } \boldsymbol{u}_{0}^{k}=\boldsymbol{u}_{0}, \\
\text { Find } \boldsymbol{u}_{n}^{k} \in \mathbb{V}_{\mathrm{div}}, \text { such that for all } \boldsymbol{v} \in \mathbb{V}_{\mathrm{div}}, \text { and } n=1,2, \ldots, N, \\
\widetilde{a}\left(\boldsymbol{u}_{n}^{k}, \boldsymbol{u}_{n}^{k}-\boldsymbol{v}\right)+\boldsymbol{\phi}\left(\boldsymbol{u}_{n}^{k}\right)-\boldsymbol{\phi}(\boldsymbol{v}) \leq \tilde{\ell}\left(\boldsymbol{u}_{n}^{k}-\boldsymbol{v}\right),
\end{array}\right.
$$

with;

$$
\begin{aligned}
\widetilde{a}(\boldsymbol{u}, \boldsymbol{v}) & =\langle\boldsymbol{u}, \boldsymbol{v}\rangle+k a(\boldsymbol{u}, \boldsymbol{v})+k b_{\boldsymbol{w}}(\boldsymbol{u}, \boldsymbol{v}) \\
\boldsymbol{\phi}(\boldsymbol{v}) & =k J(\boldsymbol{v}) \\
\widetilde{\ell}(\boldsymbol{v}) & =k\left(\boldsymbol{f}_{n}^{k}, \boldsymbol{v}\right)+\left(\boldsymbol{u}_{n-1}^{k}, \boldsymbol{v}\right) .
\end{aligned}
$$

The existence of solutions of (2.25) is standard as is suffice to show that;

(a) the bilinear and continuous form $\widetilde{a}(\cdot, \cdot)$ is coercive,

(b) $\phi(\cdot)$ is lower semi-continuous and convex,

(c) the linear form $\tilde{\ell}$ is continuous.

\section{A Priori Estimates and Convergence}

\subsection{A Priori Estimates}

In this section we derive important a priori estimates uniform with respect to the time parameter $k$ and use some compactness results, and indicate how the the solution of the solution (2.15) can be obtained in an alternative way. 
For each fixed $k$, we associate in general to the sequence $\boldsymbol{\psi}_{n}^{k}$ the following approximate functions $\boldsymbol{\psi}_{k}$ and $\widehat{\boldsymbol{\psi}}_{k}$ defined as follows for $n=1,2,3, \ldots, N$

$$
\begin{aligned}
\boldsymbol{\psi}_{k}:[0, T] & \longrightarrow \mathbb{V}_{\mathrm{div}} \\
t & \longrightarrow \boldsymbol{\psi}_{k}(t)=\boldsymbol{\psi}_{n}^{k}, t \in[(n-1) k, n k], \\
\widehat{\boldsymbol{\psi}}_{k}:[0, T] & \longrightarrow \mathbb{H} \\
t & \longrightarrow \widehat{\boldsymbol{\psi}}_{k}(t)=\frac{t-t_{n-1}}{k} \boldsymbol{\psi}_{n}^{k}+\frac{t_{n}-t}{k} \boldsymbol{\psi}_{n-1}^{k}, t \in[(n-1) k, n k] .
\end{aligned}
$$

We recall that

$$
\begin{aligned}
\left\|\boldsymbol{\psi}_{k}\right\|_{L^{2}\left(0, T ; L^{2}\right)}^{2} & =k \sum_{n=1}^{N}\left\|\boldsymbol{\psi}_{n}^{k}\right\|^{2},\left\|\boldsymbol{\psi}_{k}\right\|_{L^{\infty}\left(0, T ; L^{2}\right)}=\sup _{1 \leq n \leq N}\left\|\boldsymbol{\psi}_{n}^{k}\right\|, \\
\widehat{\boldsymbol{\psi}}_{k}^{\prime}(t) & =\delta \boldsymbol{\psi}_{n}^{k},\left\|\widehat{\boldsymbol{\psi}}_{k}^{\prime}\right\|_{L^{2}\left(0, T ; L^{2}\right)}^{2}=k \sum_{n=1}^{N}\left\|\frac{\boldsymbol{\psi}_{n}^{k}-\boldsymbol{\psi}_{n-1}^{k}}{k}\right\|^{2}, \\
2(\boldsymbol{a}-\boldsymbol{b}, \boldsymbol{a}) & =\|\boldsymbol{a}\|^{2}-\|\boldsymbol{b}\|^{2}+\|\boldsymbol{a}-\boldsymbol{b}\|^{2}, \text { for all } \boldsymbol{a}, \boldsymbol{b} \in \boldsymbol{L}^{2}(\Omega), \\
a b & \leq \frac{\epsilon}{p} a^{p}+\frac{1}{q \epsilon^{q / p}} b^{q} \text { for all } a, b, \epsilon>0, \text { with } \frac{1}{p}+\frac{1}{q}=1 .
\end{aligned}
$$

We first claim that

Lemma 3.1. Let $\left(\boldsymbol{u}_{n}^{k}\right)_{n}$ the sequence defined through (2.20). Then there exists a constant $C$ independent of $k$ such that for all $n \geq 1$,

$$
\begin{aligned}
& \left\|\boldsymbol{u}_{k}\right\|_{L^{\infty}\left(0, T ; L^{2}\right)}^{2}+\nu\left\|\nabla \boldsymbol{u}_{k}\right\|_{L^{2}\left(0, T ; L^{2}\right)}^{2}+k\left\|\widehat{\boldsymbol{u}}_{k}^{\prime}\right\|_{L^{2}\left(0, T ; L^{2}\right)}^{2}+k \sum_{n=0}^{N} J\left(\boldsymbol{u}_{k}^{n}\right) \\
& \quad \leq c\left[\left\|\boldsymbol{u}_{0}^{k}\right\|^{2}+2 k \sum_{n=1}^{N}\left(\boldsymbol{f}_{n}^{k}, \boldsymbol{u}_{n}^{k}\right)\right] .
\end{aligned}
$$

Proof. We take successively $\boldsymbol{v}=\mathbf{0}$, and $\boldsymbol{v}=2 \boldsymbol{u}_{n}^{k}$ in (2.20), using (2.12), (3.4) and adding the resulting equations, one obtains

$$
\left\|\boldsymbol{u}_{n}^{k}\right\|^{2}-\left\|\boldsymbol{u}_{n-1}^{k}\right\|^{2}+\left\|\boldsymbol{u}_{n}^{k}-\boldsymbol{u}_{n-1}^{k}\right\|^{2}+4 k \nu\left\|\varepsilon\left(\boldsymbol{u}_{n}^{k}\right)\right\|^{2}+2 k J\left(\boldsymbol{u}_{n}^{k}\right)=2 k\left(\boldsymbol{f}_{n}^{k}, \boldsymbol{u}_{n}^{k}\right) .
$$

Summing (3.7) for $n=1, \ldots, m \leq N$, one obtains

$$
\begin{aligned}
& \left\|\boldsymbol{u}_{m}^{k}\right\|^{2}+k^{2} \sum_{n=1}^{m}\left\|\delta \boldsymbol{u}_{n}^{k}\right\|^{2}+4 k \nu \sum_{n=1}^{m}\left\|\varepsilon\left(\boldsymbol{u}_{n}^{k}\right)\right\|^{2}+2 k \sum_{n=1}^{m} J\left(\boldsymbol{u}_{n}^{k}\right) \\
& =\left\|\boldsymbol{u}_{0}^{k}\right\|^{2}+2 k \sum_{n=1}^{m}\left(\boldsymbol{f}_{n}^{k}, \boldsymbol{u}_{n}^{k}\right),
\end{aligned}
$$

which implies that

$$
\begin{aligned}
& \sup _{1 \leq m \leq N}\left\|\boldsymbol{u}_{m}^{k}\right\|^{2}+k^{2} \sum_{n=1}^{N}\left\|\delta \boldsymbol{u}_{n}^{k}\right\|^{2}+4 k \nu \sum_{n=1}^{N}\left\|\varepsilon\left(\boldsymbol{u}_{n}^{k}\right)\right\|^{2}+2 k \sum_{n=1}^{N} J\left(\boldsymbol{u}_{n}^{k}\right) \\
& \quad \leq\left\|\boldsymbol{u}_{0}^{k}\right\|^{2}+2 k \sum_{n=1}^{N}\left(\boldsymbol{f}_{n}^{k}, \boldsymbol{u}_{n}^{k}\right) .
\end{aligned}
$$

Finally, applying (3.1), (3.2) and (2.8) in (3.9), one obtains the result announced. 
Corollary 3.1. If $\boldsymbol{f} \in L^{2}\left(0, T ; \boldsymbol{L}^{2}\right)$ then

$$
\left\|\boldsymbol{u}_{k}\right\|_{L^{\infty}\left(0, T ; L^{2}\right)}^{2}+\nu\left\|\nabla \boldsymbol{u}_{k}\right\|_{L^{2}\left(0, T ; L^{2}\right)}^{2}+k\left\|\widehat{\boldsymbol{u}}_{k}^{\prime}\right\|_{L^{2}\left(0, T ; L^{2}\right)}^{2} \leq C\left[\left\|\boldsymbol{u}_{0}^{k}\right\|^{2}+\|\boldsymbol{f}\|_{L^{2}\left(0, T ; L^{2}\right)}^{2}\right] .
$$

Proof. From Cauchy-Schwarz, (2.9), (3.5)

$$
\begin{aligned}
2 k \sum_{n=1}^{N}\left(\boldsymbol{f}_{n}^{k}, \boldsymbol{u}_{n}^{k}\right) & \leq 2 k \sum_{n=1}^{N}\left\|\boldsymbol{f}_{n}^{k}\right\|\left\|\boldsymbol{u}_{n}^{k}\right\| \\
& \leq 2 C_{P} k \sum_{n=1}^{N}\left\|\boldsymbol{f}_{n}^{k}\right\|\left\|\nabla \boldsymbol{u}_{n}^{k}\right\| \\
& \leq 2 C_{P} k\left[\sum_{n=1}^{N}\left\|\boldsymbol{f}_{n}^{k}\right\|^{2}\right]^{1 / 2}\left[\sum_{n=1}^{N}\left\|\nabla \boldsymbol{u}_{n}^{k}\right\|^{2}\right]^{1 / 2} \\
& \leq \frac{C_{P}^{2}}{\epsilon} k \sum_{n=1}^{N}\left\|\boldsymbol{f}_{n}^{k}\right\|^{2}+k \epsilon \sum_{n=1}^{N}\left\|\nabla \boldsymbol{u}_{n}^{k}\right\|^{2}
\end{aligned}
$$

But one quickly observe

$$
k\left\|\boldsymbol{f}_{n}^{k}\right\|^{2} \leq \int_{t_{n-1}}^{t_{n}}\|\boldsymbol{f}(t)\|^{2} d t
$$

Hence (3.10) becomes

$$
2 k \sum_{n=1}^{N}\left(\boldsymbol{f}_{n}^{k}, \boldsymbol{u}_{n}^{k}\right) \leq \frac{C_{P}^{2}}{\epsilon}\|\boldsymbol{f}\|_{L^{2}\left(0, T ; L^{2}\right)}^{2}+\epsilon\left\|\nabla \boldsymbol{u}_{k}\right\|_{L^{2}\left(0, T ; L^{2}\right)}^{2} .
$$

Returning to (3.6) with (3.11) and appropriate choice of $\epsilon$, one obtains the desired inequality.

\subsection{Passage to the Limit and Convergence}

A direct computations gives

$$
\begin{aligned}
\left\|\boldsymbol{u}_{k}-\widehat{\boldsymbol{u}}_{k}\right\|_{L^{\infty}\left(0, T ; L^{2}\right)} & =\max _{0 \leq n \leq N-1}\left\|\boldsymbol{u}_{n+1}^{k}-\boldsymbol{u}_{n}^{k}\right\|=k\left\|\widehat{\boldsymbol{u}}_{k}^{\prime}\right\|_{L^{\infty}\left(0, T ; L^{2}\right)}, \\
\left\|\boldsymbol{u}_{k}-\widehat{\boldsymbol{u}}_{k}\right\|_{L^{2}\left(0, T ; L^{2}\right)}^{2} & =\frac{k}{3} \sum_{n=0}^{N-1}\left\|\boldsymbol{u}_{n+1}^{k}-\boldsymbol{u}_{n}^{k}\right\|^{2}=\frac{k}{3}\left\|\widehat{\boldsymbol{u}}_{k}^{\prime}\right\|_{L^{2}\left(0, T ; L^{2}\right)}^{2} .
\end{aligned}
$$

Now, Corollary 3.1, and (3.13) imply that

$$
\left\|\boldsymbol{u}_{k}-\widehat{\boldsymbol{u}}_{k}\right\|_{L^{2}\left(0, T ; L^{2}\right)} \leq C k^{1 / 2}
$$

But from Corollary 3.1, there is a subsequence denoted again $\left(\boldsymbol{u}_{k}\right)_{k}$, such that

$$
\begin{aligned}
\boldsymbol{u}_{k} \longrightarrow \boldsymbol{u} & \text { in } L^{2}\left(0, T ; \mathbb{V}_{\text {div }}\right) \text { weakly, } \\
& \text { in } L^{\infty}(0, T ; \mathbb{H}) \text { weak-star. }
\end{aligned}
$$

We can extract a subsequence of $\widehat{\boldsymbol{u}}_{k}$, denoted again by $\widehat{\boldsymbol{u}}_{k}$ such that

$$
\begin{array}{ccl}
\widehat{\boldsymbol{u}}_{k} \longrightarrow \boldsymbol{w} \quad \text { in } & L^{2}\left(0, T ; \mathbb{V}_{\text {div }}\right) \text { weakly, } \\
& \text { in } & L^{\infty}(0, T ; \mathbb{H}) \text { weak-star, } \\
\widehat{\boldsymbol{u}}_{k}^{\prime} \longrightarrow \boldsymbol{w}^{\prime} \text { weakly in } & L^{2}\left(0, T ; \mathbb{V}^{\prime}\right) .
\end{array}
$$


But from (3.14), one deduces that $\boldsymbol{w}=\boldsymbol{u}$. Finally, because of the compact embedding of $\boldsymbol{H}^{1}(\Omega)$ into $\boldsymbol{L}^{2}(\Omega)$, one has:

$$
\begin{aligned}
& \widehat{\boldsymbol{u}}_{k} \longrightarrow \boldsymbol{u} \text { in } L^{2}(0, T ; \mathbb{H}) \text { strong, } \\
& \boldsymbol{u}_{k} \longrightarrow \boldsymbol{u} \text { in } L^{2}(0, T ; \mathbb{H}) \text { strong. }
\end{aligned}
$$

Next, since $\boldsymbol{f}_{k} \rightarrow \boldsymbol{f}$ in $L^{2}\left(0, T ; L^{2}\right)$ and the fact that

$$
J(\boldsymbol{u}) \leq \lim _{k \rightarrow 0} \inf J\left(\boldsymbol{u}_{k}\right),
$$

because $J$ is lower semi-continuous, one can pass to the limit in $(2.20)$ (see $[1,19])$ and obtain the following result

Theorem 3.1. Given $\boldsymbol{f} \in L^{2}(0, T ; \mathbb{H}), g \in L^{2}(S)$, and $\boldsymbol{u}_{0} \in \mathbb{H}$, there exists $\boldsymbol{u}$ satisfying;

$$
\boldsymbol{u} \in L^{2}\left(0, T ; \mathbb{V}_{\text {div }}\right) \cap L^{\infty}(0, T ; \mathbb{H}), \boldsymbol{u}^{\prime} \in L^{2}\left(0, T ; \mathbb{V}_{\text {div }}^{\prime}\right),
$$

and such that

$$
\left\{\begin{array}{l}
\boldsymbol{u}(0)=\boldsymbol{u}_{0} \text { in } \Omega, \\
\text { and for all } \boldsymbol{v} \in \mathbb{V}_{\mathrm{div}}, \text { and a.e } t \in(0, T) \\
\left\langle\boldsymbol{u}^{\prime}(t), \boldsymbol{u}(t)-\boldsymbol{v}\right\rangle+a(\boldsymbol{u}(t), \boldsymbol{u}(t)-\boldsymbol{v})+b_{\boldsymbol{w}}(\boldsymbol{u}(t), \boldsymbol{u}(t)-\boldsymbol{v}) \\
\quad+J(\boldsymbol{u}(t))-J(\boldsymbol{v}) \leq \ell(\boldsymbol{u}(t)-\boldsymbol{v})
\end{array}\right.
$$

The solutions of (2.20) and (3.18) are related in the following way.

Theorem 3.2. Let $\boldsymbol{u}$ be the solution of (3.18), and $\left(\boldsymbol{u}_{k}^{n}\right)$ the sequence solution $(2.20)$, with $\boldsymbol{f}_{k} \in L^{2}\left(0, T ; L^{2}\right)$ and $\boldsymbol{u}_{0}^{k}$ an element of $\boldsymbol{L}^{2}(\Omega)$. Then

$$
\lim _{k \rightarrow 0} \boldsymbol{u}_{k}=\boldsymbol{u} \text { strongly in } L^{2}(0, T ; \mathbb{V}) .
$$

Proof. First we have

$$
\frac{1}{2} \frac{d}{d t}\left\|\boldsymbol{u}-\widehat{\boldsymbol{u}}_{k}\right\|^{2}=\left\langle\boldsymbol{u}^{\prime}, \boldsymbol{u}-\widehat{\boldsymbol{u}}_{k}\right\rangle-\left\langle\widehat{\boldsymbol{u}}_{k}^{\prime}, \boldsymbol{u}-\widehat{\boldsymbol{u}}_{k}\right\rangle .
$$

We take $\boldsymbol{v}=\widehat{\boldsymbol{u}}_{k}$ in (3.18) and $\boldsymbol{v}=\boldsymbol{u}$ in (2.20), adding the resulting inequalities leads to

$$
\begin{aligned}
\left\langle\boldsymbol{u}^{\prime}, \boldsymbol{u}-\widehat{\boldsymbol{u}}_{k}\right\rangle-\left\langle\widehat{\boldsymbol{u}}_{k}^{\prime}, \boldsymbol{u}-\widehat{\boldsymbol{u}}_{k}\right\rangle \leq & a\left(\boldsymbol{u}, \widehat{\boldsymbol{u}}_{k}-\boldsymbol{u}_{k}\right)-a\left(\boldsymbol{u}-\boldsymbol{u}_{k}, \boldsymbol{u}-\boldsymbol{u}_{k}\right) \\
& +b_{\boldsymbol{w}}\left(\boldsymbol{u}, \widehat{\boldsymbol{u}}_{k}-\boldsymbol{u}\right)+b_{\boldsymbol{w}}\left(\boldsymbol{u}_{k}, \boldsymbol{u}-\boldsymbol{u}_{k}\right) \\
& +J\left(\widehat{\boldsymbol{u}}_{k}\right)-J\left(\boldsymbol{u}_{k}\right)+\left(\boldsymbol{f}, \boldsymbol{u}_{k}-\widehat{\boldsymbol{u}}_{k}\right)+\left(\boldsymbol{f}_{k}-\boldsymbol{f}, \boldsymbol{u}_{k}-\boldsymbol{u}\right) \\
& +\left(\widehat{\boldsymbol{u}}_{k}^{\prime}, \widehat{\boldsymbol{u}}_{k}-\boldsymbol{u}_{k}\right) .
\end{aligned}
$$

From the convexity of $J$, we find that

$$
\begin{aligned}
J\left(\widehat{\boldsymbol{u}}_{k}\right)-J\left(\boldsymbol{u}_{n}^{k}\right) & =J\left(\frac{t-t_{n-1}}{k} \boldsymbol{u}_{n}^{k}+\frac{t_{n}-t}{k} \boldsymbol{u}_{n-1}^{k}\right)-J\left(\boldsymbol{u}_{n}^{k}\right) \\
& \leq\left(t-t_{n}\right) \frac{J\left(\boldsymbol{u}_{n}^{k}\right)-J\left(\boldsymbol{u}_{n-1}^{k}\right)}{k} \equiv\left(t-t_{n}\right) \delta J\left(\boldsymbol{u}_{k}\right) .
\end{aligned}
$$

Now, inserting (3.20) in (3.19) and having in mind (3.21) and (2.12) we obtain

$$
\begin{aligned}
\frac{1}{2} \frac{d}{d t}\left\|\boldsymbol{u}-\widehat{\boldsymbol{u}}_{k}\right\|^{2}+a\left(\boldsymbol{u}-\boldsymbol{u}_{k}, \boldsymbol{u}-\boldsymbol{u}_{k}\right) \leq & a\left(\boldsymbol{u}, \widehat{\boldsymbol{u}}_{k}-\boldsymbol{u}_{k}\right)+b_{\boldsymbol{w}}\left(\boldsymbol{u}, \widehat{\boldsymbol{u}}_{k}-\boldsymbol{u}_{k}\right)+\left(t-t_{n}\right) \delta J\left(\boldsymbol{u}_{k}\right) \\
& +\left(\boldsymbol{f}, \boldsymbol{u}_{k}-\widehat{\boldsymbol{u}}_{k}\right)+\left(\boldsymbol{f}_{k}-\boldsymbol{f}, \boldsymbol{u}_{k}-\boldsymbol{u}\right) \\
& +\left(\widehat{\boldsymbol{u}}_{k}^{\prime}, \widehat{\boldsymbol{u}}_{k}-\boldsymbol{u}_{k}\right) .
\end{aligned}
$$


A direct computations reveals that

$$
\begin{aligned}
\widehat{\boldsymbol{u}}_{k}-\boldsymbol{u}_{k} & =\left(t-t_{n}\right) \widehat{\boldsymbol{u}}_{k}^{\prime}=\left(t-t_{n}\right) \delta \boldsymbol{u}_{k}, \\
\left\langle\widehat{\boldsymbol{u}}_{k}^{\prime}, \widehat{\boldsymbol{u}}_{k}-\boldsymbol{u}_{k}\right\rangle & =\left(t-t_{n}\right)\left\|\widehat{\boldsymbol{u}}_{k}^{\prime}\right\|^{2} .
\end{aligned}
$$

Thus (3.22) becomes

$$
\begin{aligned}
\frac{1}{2} \frac{d}{d t}\left\|\boldsymbol{u}-\widehat{\boldsymbol{u}}_{k}\right\|^{2}+a\left(\boldsymbol{u}-\boldsymbol{u}_{k}, \boldsymbol{u}-\boldsymbol{u}_{k}\right) \leq & a\left(\boldsymbol{u}, \widehat{\boldsymbol{u}}_{k}-\boldsymbol{u}_{k}\right)+b_{\boldsymbol{w}}\left(\boldsymbol{u}, \widehat{\boldsymbol{u}}_{k}-\boldsymbol{u}_{k}\right)+\left(t-t_{n}\right) \delta J\left(\boldsymbol{u}_{k}\right) \\
& -\left(\boldsymbol{f}, \widehat{\boldsymbol{u}}_{k}-\boldsymbol{u}_{k}\right)+\left(\boldsymbol{f}_{k}-\boldsymbol{f}, \boldsymbol{u}_{k}-\boldsymbol{u}\right) \\
& +\left(t-t_{n}\right)\left\|\delta \boldsymbol{u}_{k}\right\|^{2} .
\end{aligned}
$$

We integrate $(3.23)$ over $\left(t_{n-1}, t_{n}\right)$ and we take the sum for $n=1,2, \ldots, m$. We find (dropping some positive terms)

$$
\begin{aligned}
\int_{0}^{t_{m}} a\left(\boldsymbol{u}-\boldsymbol{u}_{k}, \boldsymbol{u}-\boldsymbol{u}_{k}\right) d t \leq & \int_{0}^{t_{m}} a\left(\boldsymbol{u}, \widehat{\boldsymbol{u}}_{k}-\boldsymbol{u}_{k}\right) d t+\int_{0}^{t_{m}} b_{\boldsymbol{w}}\left(\boldsymbol{u}, \widehat{\boldsymbol{u}}_{k}-\boldsymbol{u}_{k}\right) d t+\frac{k}{2} J\left(\boldsymbol{u}_{0}^{k}\right) \\
& -\int_{0}^{t_{m}}\left(\boldsymbol{f}, \widehat{\boldsymbol{u}}_{k}-\boldsymbol{u}_{k}\right) d t+\int_{0}^{t_{m}}\left(\boldsymbol{f}_{k}-\boldsymbol{f}, \boldsymbol{u}_{k}-\boldsymbol{u}\right) d t
\end{aligned}
$$

The strong convergence of $\boldsymbol{f}_{n}^{k}$ to $\boldsymbol{f}$, the weak convergence of $\boldsymbol{u}_{k}$ to $\boldsymbol{u}$, the strong convergence of $\widehat{\boldsymbol{u}}_{k}-\boldsymbol{u}_{k}$ to zero in $L^{2}(0, T ; \mathbb{V})$, and the strong convergence of $\boldsymbol{u}_{k}-\boldsymbol{u}$ to zero in $L^{2}(0, T ; \mathbb{H})$ and the Korn's inequality yield the convergence announced in theorem 3.2 .

\section{Error Estimates}

The goal of this paragraph is to estimate the difference $\boldsymbol{u}-\widehat{\boldsymbol{u}}_{k}$.

To present the next result, we first define $\widetilde{\boldsymbol{u}}_{k}$ as

$$
\widetilde{\boldsymbol{\psi}}_{k}(t)=\frac{1}{k} \int_{t}^{t+k} \widehat{\boldsymbol{\psi}}_{k}(s) d s
$$

The chain's rule formula leads to

$$
\widetilde{\boldsymbol{\psi}}_{k}^{\prime}(t)=\frac{\widehat{\boldsymbol{\psi}}_{k}(t+k)-\widehat{\boldsymbol{\psi}}_{k}(t)}{k}, \quad \widetilde{\boldsymbol{\psi}}_{k}^{\prime \prime}(t)=\frac{\widehat{\boldsymbol{\psi}}_{k}^{\prime}(t+k)-\widehat{\boldsymbol{\psi}}_{k}^{\prime}(t)}{k} .
$$

We claim that:

Lemma 4.1. Let $\left(\boldsymbol{u}_{n}^{k}\right)_{n}$ be the sequence defined through (2.20). Then there exists a constant $C$ independent of $k$ such that for all $n \geq 1$,

$$
\begin{aligned}
& \left\|\widehat{\boldsymbol{u}}_{k}^{\prime}\right\|_{L^{\infty}\left(0, T ; L^{2}\right)}^{2}+\nu\left\|\nabla \widehat{\boldsymbol{u}}_{k}^{\prime}\right\|_{L^{2}\left(0, T ; L^{2}\right)}^{2}+k\left\|\widetilde{\boldsymbol{u}}_{k}^{\prime \prime}\right\|_{L^{2}\left(0, T ; L^{2}\right)}^{2} \\
& \quad \leq C\left[\left\|\delta \boldsymbol{u}_{0}^{k}\right\|^{2}+2 k \sum_{n=1}^{m}\left(\delta \boldsymbol{f}_{n}^{k}, \delta \boldsymbol{u}_{n}^{k}\right)\right]
\end{aligned}
$$

Before embarking on the proof of Lemma 4.1, it is important to mention that for the definition of $\delta \boldsymbol{u}_{0}^{k}$, there are two possibilities; one can take $\boldsymbol{u}_{-1}^{k}=\boldsymbol{u}_{0}^{k}$ so that $\delta \boldsymbol{u}_{0}^{k}=0$, otherwise we choose $\boldsymbol{u}_{-1}^{k}=\boldsymbol{u}_{0}$, and compute $\boldsymbol{u}_{0}^{k}$ in such a way that $(2.20)$ is valid for $n=0$, this is:

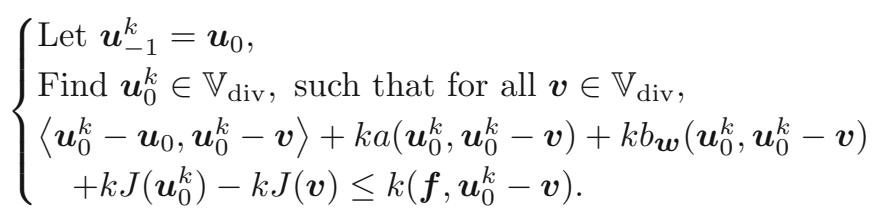


Proof of Lemma 4.1. Take $\boldsymbol{v}=\boldsymbol{u}_{n+1}^{k}$ in (2.20) gives

$$
\left\{\begin{array}{c}
\left\langle\delta \boldsymbol{u}_{n}^{k}, \boldsymbol{u}_{n}^{k}-\boldsymbol{u}_{n+1}^{k}\right\rangle+a\left(\boldsymbol{u}_{n}^{k}, \boldsymbol{u}_{n}^{k}-\boldsymbol{u}_{n+1}^{k}\right)+b_{\boldsymbol{w}}\left(\boldsymbol{u}_{n}^{k}, \boldsymbol{u}_{n}^{k}-\boldsymbol{u}_{n+1}^{k}\right) \\
+J\left(\boldsymbol{u}_{n}^{k}\right)-J\left(\boldsymbol{u}_{n+1}^{k}\right) \leq\left(\boldsymbol{f}_{n}^{k}, \boldsymbol{u}_{n}^{k}-\boldsymbol{u}_{n+1}^{k}\right) .
\end{array}\right.
$$

Writing (2.20) at the time step $t_{n+1}$, and taking in the resulting equation $\boldsymbol{v}=\boldsymbol{u}_{n}^{k}$, one obtains

$$
\left\{\begin{array}{c}
\left\langle\delta \boldsymbol{u}_{n+1}^{k}, \boldsymbol{u}_{n+1}^{k}-\boldsymbol{u}_{n}^{k}\right\rangle+a\left(\boldsymbol{u}_{n+1}^{k}, \boldsymbol{u}_{n+1}^{k}-\boldsymbol{u}_{n}^{k}\right)+b_{\boldsymbol{w}}\left(\boldsymbol{u}_{n+1}^{k}, \boldsymbol{u}_{n+1}^{k}-\boldsymbol{u}_{n}^{k}\right) \\
+J\left(\boldsymbol{u}_{n+1}^{k}\right)-J\left(\boldsymbol{u}_{n}^{k}\right) \leq\left(\boldsymbol{f}_{n+1}^{k}, \boldsymbol{u}_{n+1}^{k}-\boldsymbol{u}_{n}^{k}\right) .
\end{array}\right.
$$

Do $2 \times(4.5)+2 \times(4.6)$, using $(2.12)$, one gets

$$
2 k\left\langle\delta \boldsymbol{u}_{n+1}^{k}-\delta \boldsymbol{u}_{n}^{k}, \delta \boldsymbol{u}_{n+1}^{k}\right\rangle+2 k^{2} a\left(\delta \boldsymbol{u}_{n+1}^{k}, \delta \boldsymbol{u}_{n+1}^{k}\right) \leq 2 k^{2}\left(\delta \boldsymbol{f}_{n+1}^{k}, \delta \boldsymbol{u}_{n+1}^{k}\right) .
$$

From (3.4), we deduce that

$$
\begin{aligned}
2 k\left\langle\delta \boldsymbol{u}_{n+1}^{k}-\delta \boldsymbol{u}_{n}^{k}, \delta \boldsymbol{u}_{n+1}^{k}\right\rangle & =k\left\|\delta \boldsymbol{u}_{n+1}^{k}\right\|^{2}-k\left\|\delta \boldsymbol{u}_{n}^{k}\right\|^{2}+k\left\|\delta \boldsymbol{u}_{n+1}^{k}-\delta \boldsymbol{u}_{n}^{k}\right\|^{2} \\
& =k\left\|\delta \boldsymbol{u}_{n+1}^{k}\right\|^{2}-k\left\|\delta \boldsymbol{u}_{n}^{k}\right\|^{2}+k^{3}\left\|\delta^{2} \boldsymbol{u}_{n+1}^{k}\right\|^{2}
\end{aligned}
$$

Replacing (4.8) in the left hand side of (4.7), we have

$$
\left\|\delta \boldsymbol{u}_{n+1}^{k}\right\|^{2}+k^{2}\left\|\delta^{2} \boldsymbol{u}_{n+1}^{k}\right\|^{2}+2 k a\left(\delta \boldsymbol{u}_{n+1}^{k}, \delta \boldsymbol{u}_{n+1}^{k}\right) \leq\left\|\delta \boldsymbol{u}_{n}^{k}\right\|^{2}+2 k\left(\delta \boldsymbol{f}_{n+1}^{k}, \delta \boldsymbol{u}_{n+1}^{k}\right) .
$$

Adding (4.9) for $n=0,1,2, \ldots, m-1$; gives

$$
\left\|\delta \boldsymbol{u}_{m}^{k}\right\|^{2}+k^{2} \sum_{n=1}^{m}\left\|\delta^{2} \boldsymbol{u}_{n}^{k}\right\|^{2}+4 \nu k \sum_{n=1}^{m}\left\|\varepsilon\left(\delta \boldsymbol{u}_{n}^{k}\right)\right\|^{2} \leq\left\|\delta \boldsymbol{u}_{0}^{k}\right\|^{2}+2 k \sum_{n=1}^{m}\left(\delta \boldsymbol{f}_{n}^{k}, \delta \boldsymbol{u}_{n}^{k}\right),
$$

from which we deduce the result announced by the application of (2.8).

Corollary 4.1. If $\boldsymbol{f}^{\prime} \in L^{2}\left(0, T ; L^{2}\right)$ then

$$
\begin{aligned}
& \left\|\widehat{\boldsymbol{u}}_{k}^{\prime}\right\|_{L^{\infty}\left(0, T ; L^{2}\right)}^{2}+\nu\left\|\nabla \widehat{\boldsymbol{u}}_{k}^{\prime}\right\|_{L^{2}\left(0, T ; L^{2}\right)}^{2}+k\left\|\widetilde{\boldsymbol{u}}_{k}^{\prime \prime}\right\|_{L^{2}\left(0, T ; L^{2}\right)}^{2} \\
& \quad \leq C\left[\left\|\delta \boldsymbol{u}_{0}^{k}\right\|^{2}+\left\|\boldsymbol{f}^{\prime}\right\|_{L^{2}\left(0, T ; L^{2}\right)}^{2}\right] .
\end{aligned}
$$

Proof. We just need to bound the right hand side of the estimate obtained in Lemma 4.1. For that purpose, we successively obtain

$$
\begin{aligned}
2 k \sum_{n=1}^{m}\left(\delta \boldsymbol{f}_{n}^{k}, \delta \boldsymbol{u}_{n}^{k}\right) & \leq 2 k \sum_{n=1}^{m}\left\|\delta \boldsymbol{f}_{n}^{k}\right\|\left\|\delta \boldsymbol{u}_{n}^{k}\right\| \\
& \leq 2 C_{P} k \sum_{n=1}^{m}\left\|\delta \boldsymbol{f}_{n}^{k}\right\|\left\|\nabla \delta \boldsymbol{u}_{n}^{k}\right\| \\
& \leq \frac{C_{P}^{2}}{\epsilon} k \sum_{n=1}^{m}\left\|\delta \boldsymbol{f}_{n}^{k}\right\|^{2}+k \epsilon \sum_{n=1}^{m}\left\|\nabla \delta \boldsymbol{u}_{n}^{k}\right\|^{2} \\
& \leq \frac{C_{P}^{2}}{\epsilon}\left\|\widehat{\boldsymbol{f}}_{k}^{\prime}\right\|_{L^{2}\left(0, T ; L^{2}\right)}^{2}+\epsilon\left\|\nabla \widehat{\boldsymbol{u}}_{k}^{\prime}\right\|_{L^{2}\left(0, T ; L^{2}\right)}^{2} \\
& \leq \frac{C_{P}^{2}}{\epsilon}\left\|\boldsymbol{f}^{\prime}\right\|_{L^{2}\left(0, T ; L^{2}\right)}^{2}+\epsilon\left\|\nabla \widehat{\boldsymbol{u}}_{k}^{\prime}\right\|_{L^{2}\left(0, T ; L^{2}\right)}^{2}
\end{aligned}
$$

which together with an appropriate choice of $\epsilon$ and (4.3) gives the desired inequality.

The main result of the section, is the following: 
Theorem 4.1. Assume that $\boldsymbol{f} \in \boldsymbol{H}^{1}\left(0, T ; L^{2}\right)$, let $\boldsymbol{u}$ be the solution of (3.18), and $\left(\boldsymbol{u}_{k}^{n}\right)_{n}$ the sequence of solution of (2.20), with $\delta \boldsymbol{u}_{0}^{k}$ an element of $\boldsymbol{L}^{2}(\Omega)$. Then there is $C$, independent of $k$ such that

$$
\begin{aligned}
\sum_{n=1}^{N}\left\|\boldsymbol{u}(t)-\widehat{\boldsymbol{u}}_{k}(t)\right\|^{2} \leq & C k^{2}\left(\left\|\delta \boldsymbol{u}_{0}^{k}\right\|^{2}+\left\|\boldsymbol{f}^{\prime}\right\|_{L^{2}\left(0, T ; L^{2}\right)}^{2}\right)+C k^{2} \sum_{n=1}^{N} \mathcal{E}_{n} \\
& +C k^{2}\left\|\boldsymbol{f}^{\prime}\right\|_{L^{2}\left(0, T ; L^{2}\right)}^{2} \\
\left\|\nabla\left(\boldsymbol{u}-\widehat{\boldsymbol{u}}_{k}\right)\right\|_{L^{2}\left(0, T ; L^{2}\right)}^{2} \leq & C \sum_{n=1}^{N}\left\|\boldsymbol{u}(t)-\widehat{\boldsymbol{u}}_{k}(t)\right\|^{2}+C k^{2}\left(\left\|\delta \boldsymbol{u}_{0}^{k}\right\|^{2}+\left\|\boldsymbol{f}^{\prime}\right\|_{L^{2}\left(0, T ; L^{2}\right)}^{2}\right) \\
& +C k^{2}\left\|\boldsymbol{f}^{\prime}\right\|_{L^{2}\left(0, T ; L^{2}\right)}^{2}+k^{2} \sum_{n=1}^{N} \mathcal{E}_{n} .
\end{aligned}
$$

Proof. Recall that (3.22) can also be re-written as

$$
\begin{aligned}
\frac{1}{2} \frac{d}{d t}\left\|\boldsymbol{u}-\widehat{\boldsymbol{u}}_{k}\right\|^{2}+a\left(\boldsymbol{u}-\boldsymbol{u}_{n}^{k}, \boldsymbol{u}-\boldsymbol{u}_{n}^{k}\right) \leq & \left(t-t_{n}\right) a\left(\boldsymbol{u}, \widehat{\boldsymbol{u}}_{k}^{\prime}\right)+\left(t-t_{n}\right) b_{\boldsymbol{w}}\left(\boldsymbol{u}, \widehat{\boldsymbol{u}}_{k}^{\prime}\right)+\left(t-t_{n}\right) \delta J\left(\boldsymbol{u}_{n}^{k}\right) \\
& -\left(t-t_{n}\right)\left(\boldsymbol{f}, \widehat{\boldsymbol{u}}_{k}^{\prime}\right)+\left(\boldsymbol{f}_{n}^{k}-\boldsymbol{f}, \boldsymbol{u}_{n}^{k}-\boldsymbol{u}\right) \\
& +\left(t-t_{n}\right)\left\langle\widehat{\boldsymbol{u}}_{k}^{\prime}, \widehat{\boldsymbol{u}}_{k}^{\prime}\right\rangle
\end{aligned}
$$

Next, $\boldsymbol{v}=\boldsymbol{u}_{n-1}^{k}$ in (2.20) gives

$$
-\mathcal{E}_{n}=\left\langle\widehat{\boldsymbol{u}}_{k}^{\prime}, \widehat{\boldsymbol{u}}_{k}^{\prime}\right\rangle+a\left(\boldsymbol{u}_{n}^{k}, \widehat{\boldsymbol{u}}_{k}^{\prime}\right)+b_{\boldsymbol{w}}\left(\boldsymbol{u}_{n}^{k}, \widehat{\boldsymbol{u}}_{k}^{\prime}\right)+\delta J\left(\boldsymbol{u}_{n}^{k}\right)-\left(\boldsymbol{f}_{n}^{k}, \widehat{\boldsymbol{u}}_{k}^{\prime}\right) \leq 0 .
$$

Inserting (4.13) in (4.12) and using the linearity of $a(\cdot, \cdot)$ and (2.12), the inequalities of Cauchy-Shwarz together with (2.9), (2.8) yield

$$
\begin{aligned}
\frac{1}{2} \frac{d}{d t}\left\|\boldsymbol{u}-\widehat{\boldsymbol{u}}_{k}\right\|^{2}+a\left(\boldsymbol{u}-\widehat{\boldsymbol{u}}_{k}, \boldsymbol{u}-\widehat{\boldsymbol{u}}_{k}\right) \leq & \left(t-t_{n}\right) a\left(\widehat{\boldsymbol{u}}_{k}-\boldsymbol{u}, \widehat{\boldsymbol{u}}_{k}^{\prime}\right)+\left(t-t_{n}\right) b_{\boldsymbol{w}}\left(\boldsymbol{u}-\widehat{\boldsymbol{u}}_{k}, \widehat{\boldsymbol{u}}_{k}^{\prime}\right) \\
& +\left(\boldsymbol{f}_{n}^{k}-\boldsymbol{f}, \widehat{\boldsymbol{u}}_{k}-\boldsymbol{u}\right)-\left(t-t_{n}\right) \mathcal{E}_{n} \\
\leq & 2 \nu k\left\|\varepsilon\left(\widehat{\boldsymbol{u}}_{k}-\boldsymbol{u}\right)\right\|\left\|\varepsilon\left(\widehat{\boldsymbol{u}}_{k}^{\prime}\right)\right\|+k \frac{\|b \boldsymbol{w}\|}{C_{K}} \| \varepsilon\left(\boldsymbol{u}-\widehat{\boldsymbol{u}}_{k}\|\| \varepsilon\left(\widehat{\boldsymbol{u}}_{k}^{\prime}\right) \|\right. \\
& +\frac{1}{C_{K}^{1 / 2} C_{P}^{1 / 2}}\left\|\boldsymbol{f}_{n}^{k}-\boldsymbol{f}\right\|\left\|\varepsilon\left(\widehat{\boldsymbol{u}}_{k}-\boldsymbol{u}\right)\right\|+k \mathcal{E}_{n},
\end{aligned}
$$

which by (3.5) leads to

$$
\begin{aligned}
\frac{1}{2} \frac{d}{d t}\left\|\boldsymbol{u}-\widehat{\boldsymbol{u}}_{k}\right\|^{2}+\frac{\nu}{2}\left\|\varepsilon\left(\boldsymbol{u}-\widehat{\boldsymbol{u}}_{k}\right)\right\|^{2} \leq & k^{2}\left(\nu+2 \frac{\left\|b_{\boldsymbol{w}}\right\|^{2}}{\nu C_{K}^{2}}\right)\left\|\varepsilon\left(\widehat{\boldsymbol{u}}_{k}^{\prime}\right)\right\|^{2} \\
& +\frac{2}{\nu C_{K} C_{P}}\left\|\boldsymbol{f}_{n}^{k}-\boldsymbol{f}\right\|^{2}+k \mathcal{E}_{n} .
\end{aligned}
$$

Applying again (2.8) and (2.9), (4.14) become

$$
\begin{aligned}
\frac{d}{d t}\left\|\boldsymbol{u}-\widehat{\boldsymbol{u}}_{k}\right\|^{2}+\nu C_{K} C_{P}\left\|\boldsymbol{u}-\widehat{\boldsymbol{u}}_{k}\right\|^{2} \leq & 2 k^{2}\left[\nu+2 \frac{\left\|b_{\boldsymbol{w}}\right\|^{2}}{\nu C_{K}^{2}}\right]\left\|\nabla \delta \boldsymbol{u}_{k}^{n}\right\|^{2} \\
& +\frac{4}{\nu C_{K} C_{P}}\left\|\boldsymbol{f}_{n}^{k}-\boldsymbol{f}\right\|^{2}+2 k \mathcal{E}_{n} .
\end{aligned}
$$

Integration of the differential inequality (4.15) and taking the summation for $n=1,2, \ldots, N$, one obtains

$$
\begin{aligned}
\sum_{n=1}^{N}\left\|\boldsymbol{u}(t)-\widehat{\boldsymbol{u}}_{k}(t)\right\|^{2} \leq & \frac{2 k^{2}}{\nu C_{K} C_{P}}\left[\nu+2 \frac{\left\|b_{\boldsymbol{w}}\right\|^{2}}{\nu C_{K}^{2}}\right]\left\|\nabla \widehat{\boldsymbol{u}}_{k}^{\prime}\right\|_{L^{2}\left(0, T ; L^{2}\right)}^{2}+\frac{2 k^{2}}{\nu C_{K} C_{P}} \sum_{n=1}^{N} \mathcal{E}_{n} \\
& +\frac{4}{\nu C_{K} C_{P}}\left\|\boldsymbol{f}_{k}-\boldsymbol{f}\right\|_{L^{2}\left(0, T ; L^{2}\right)}^{2},
\end{aligned}
$$


which leads to (4.10) after application of Corollary 4.1, and error estimate

$$
\left\|\boldsymbol{f}_{n}^{k}-\boldsymbol{f}\right\|_{L^{2}\left(t_{n-1}, t_{n} ; L^{2}\right)} \leq C k\left\|\boldsymbol{f}^{\prime}\right\|_{L^{2}\left(t_{n-1}, t_{n} ; L^{2}\right)} .
$$

Next, integrating (4.14) over $\left(t_{n-1}, t_{n}\right)$, using (2.8), one obtains

$$
\begin{aligned}
\int_{t_{n-1}}^{t_{n}}\left\|\nabla\left(\boldsymbol{u}-\widehat{\boldsymbol{u}}_{k}\right)\right\|^{2} d s \leq & C\left\|\boldsymbol{u}\left(t_{n-1}\right)-\widehat{\boldsymbol{u}}_{k}\left(t_{n-1}\right)\right\|^{2}+C k^{3}\left\|\nabla \delta \boldsymbol{u}_{n}^{k}\right\|^{2} \\
& +\int_{t_{n-1}}^{t_{n}}\left\|\boldsymbol{f}_{n}^{k}-\boldsymbol{f}(s)\right\|^{2} d s+k^{2} \mathcal{E}_{n},
\end{aligned}
$$

from which we easily deduce that

$$
\begin{aligned}
\left\|\nabla\left(\boldsymbol{u}-\widehat{\boldsymbol{u}}_{k}\right)\right\|_{L^{2}\left(0, T ; L^{2}\right)}^{2} \leq & C \sum_{n=1}^{N}\left\|\boldsymbol{u}(t)-\widehat{\boldsymbol{u}}_{k}(t)\right\|^{2}+C k^{2}\left\|\nabla \widehat{\boldsymbol{u}}_{k}^{\prime}\right\|_{L^{2}\left(0, T ; L^{2}\right)}^{2} \\
& +C\left\|\boldsymbol{f}_{k}-\boldsymbol{f}\right\|_{L^{2}\left(0, T ; L^{2}\right)}^{2}+k^{2} \sum_{n=1}^{N} \mathcal{E}_{n}
\end{aligned}
$$

Finally, one gets (4.11) after application of (4.16) and Corollary 4.1 in (4.18).

It is manifest that to close the error estimates in Theorem 4.1, one needs to estimates $\sum_{n=1}^{N} \mathcal{E}_{n}$. For that purpose, we claim that

Lemma 4.2. Assume that $\boldsymbol{f} \in \boldsymbol{H}^{1}\left(0, T ; L^{2}\right)$, let $\left(\boldsymbol{u}_{k}^{n}\right)_{n}$ the sequence of solution of $(2.20)$, with $\delta \boldsymbol{u}_{0}^{k}$ an element of $\boldsymbol{L}^{2}(\Omega)$. Then there is $C$, independent of $k$ such that

$$
\sum_{n=1}^{N} \mathcal{E}_{n} \leq C\left\|\delta \boldsymbol{u}_{0}^{k}\right\|^{2}+C\left\|\boldsymbol{f}^{\prime}\right\|_{L^{2}\left(0, T ; L^{2}\right)}^{2} .
$$

Proof. We recall that (4.13) can be re-written as

$$
\mathcal{E}_{n}=-\left\langle\delta \boldsymbol{u}_{n}^{k}, \delta \boldsymbol{u}_{n}^{k}\right\rangle-a\left(\boldsymbol{u}_{n}^{k}, \delta \boldsymbol{u}_{n}^{k}\right)-b_{\boldsymbol{w}}\left(\boldsymbol{u}_{n}^{k}, \delta \boldsymbol{u}_{n}^{k}\right)-\delta J\left(\boldsymbol{u}_{n}^{k}\right)+\left(\boldsymbol{f}_{n}^{k}, \delta \boldsymbol{u}_{n}^{k}\right) .
$$

We take the Eq. (2.20) in time step $n-1$ and replace $\boldsymbol{v}$ by $\boldsymbol{u}_{n}^{k}$. One obtains

$$
-\delta J\left(\boldsymbol{u}_{n}^{k}\right) \leq\left\langle\delta \boldsymbol{u}_{n-1}^{k}, \delta \boldsymbol{u}_{n}^{k}\right\rangle+a\left(\boldsymbol{u}_{n-1}^{k}, \delta \boldsymbol{u}_{n}^{k}\right)+b_{\boldsymbol{w}}\left(\boldsymbol{u}_{n-1}^{k}, \delta \boldsymbol{u}_{n}^{k}\right)-\left(\boldsymbol{f}_{n-1}^{k}, \delta \boldsymbol{u}_{n}^{k}\right) .
$$

(4.21) in (4.20) gives

$$
\begin{aligned}
\mathcal{E}_{n} & \leq-\frac{1}{2}\left\|\delta \boldsymbol{u}_{n}^{k}\right\|+\frac{1}{2}\left\|\delta \boldsymbol{u}_{n-1}^{k}\right\|^{2}-\frac{1}{2}\left\|\delta \boldsymbol{u}_{n}^{k}-\delta \boldsymbol{u}_{n-1}^{k}\right\|^{2}+k a\left(\delta \boldsymbol{u}_{n}^{k}, \delta \boldsymbol{u}_{n}^{k}\right)+k\left(\delta \boldsymbol{f}_{n}^{k}, \delta \boldsymbol{u}_{n}^{k}\right) \\
& \leq-\frac{1}{2}\left\|\delta \boldsymbol{u}_{n}^{k}\right\|+\frac{1}{2}\left\|\delta \boldsymbol{u}_{n-1}^{k}\right\|^{2}+2 \nu k\left\|\nabla \delta \boldsymbol{u}_{n}^{k}\right\|^{2}+k C_{P}^{1 / 2}\left\|\delta \boldsymbol{f}_{n}^{k}\right\|\left\|\nabla \delta \boldsymbol{u}_{n}^{k}\right\|,
\end{aligned}
$$

which by summation for $n=1,2, \ldots, N$, and (3.5) yields

$$
\begin{aligned}
\sum_{n=1}^{N} \mathcal{E}_{n} & \leq-\frac{1}{2}\left\|\delta \boldsymbol{u}_{N}^{k}\right\|^{2}+\frac{1}{2}\left\|\delta \boldsymbol{u}_{0}^{k}\right\|^{2}+2 \nu k \sum_{n=1}^{N}\left\|\nabla \delta \boldsymbol{u}_{n}^{k}\right\|^{2}+k C_{P}^{1 / 2} \sum_{n=1}^{N}\left\|\delta \boldsymbol{f}_{n}^{k}\right\|\left\|\nabla \delta \boldsymbol{u}_{n}^{k}\right\| \\
& \leq-\frac{1}{2}\left\|\delta \boldsymbol{u}_{N}^{k}\right\|^{2}+\frac{1}{2}\left\|\delta \boldsymbol{u}_{0}^{k}\right\|^{2}+C\left\|\nabla \widehat{\boldsymbol{u}}_{k}^{\prime}\right\|_{L^{2}\left(0, T ; L^{2}\right)}^{2}+C\left\|\boldsymbol{f}^{\prime}\right\|_{L^{2}\left(0, T ; L^{2}\right)}^{2} .
\end{aligned}
$$

We deduce the result announced by application of Corollary 4.1

Remark 4.1. From Lemma 4.2 and Theorem 4.1, it is manifest that $\left\|\nabla\left(\boldsymbol{u}-\widehat{\boldsymbol{u}}_{k}\right)\right\|_{L^{2}\left(0, T ; L^{2}\right)}$ and $\| \boldsymbol{u}(t)-$ $\widehat{\boldsymbol{u}}_{k}(t) \|$ for any $t \in(0, T]$ are of order one. 


\section{References}

[1] Temam, R.: Navier Stokes Equations: Theory and Numerical Analysis, 2nd edn. AMS Chelsea Publishing, New York (2001)

[2] Ju, N.: On the global stability of a temporal discretization scheme for the Navier Stokes equations. IMA J. Numer. Anal. 22, 577-597 (2002)

[3] Tone, F., Wirosetisno, D.: On the long time stability of the implicit euler scheme for two-dimensional Navier-Stokes equations. Siam J. Numer. Anal. 44, 29-40 (2006)

[4] Tone, F.: On the long time $H^{2}$ stability of the implicit Euler scheme for two-dimensional magnetohydrodynamic equations. J. Sci. Comput. 38, 331-348 (2009)

[5] Ewald, B., Tone, F.: Approximation of the long term dynamics of the dynamical system generated by the two dimensional thermohydraulics equations. Int. J. Numer. Anal. Model. 10(3), 509-535 (2013)

[6] Nochetto, R., Savare, G., Verdi, C.: A posteriori error estimates for variable time-step discretizations of nonlinear evolution equations. Commun. Pure Appl. Math. 53(5), 525-589 (2000)

[7] Rulla, J.: Error analysis for implicit approximations to solutions to Cauchy problems. Siam J. Numer. Anal. 33, 68$87(1996)$

[8] Li, Y., Li, K.: Semi-discrete finite element methods for Navier Stokes equations with nonlinear slip boundary conditions based on regularization procedure. Numer. Math. 117, 1-36 (2011)

[9] Fujita, H., Kawarada, H.: Variational inequalities for the Stokes equation with boundary conditions of friction type. In: Recent Development in Domain Decomposition Methods and Flow Problems. Gakuto International series of Mathematical sciences and its applications, vol. 11, pp. 15-33 (1998)

[10] Fujita, H.: A mathematical analysis of motions of viscous incompressible fluid under leak or slip boundary conditions. In: Mathematical Fluid Mechanics and Modeling (Kyoto, 1994), RIMS Kōkyūroko, vol. 888, pp. 199-216. Kyoto University, Kyoto (1994)

[11] Fujita, H.: Non-stationary Stokes flows under leak boundary conditions of friction type. J. Comput. Appl. Math. 19, 1$8(2001)$

[12] Le Roux, C.: Steady Stokes flows with threshold boundary conditions. Math. Models Methods Appl. Sci. 15(8), 1141$1168(2005)$

[13] Le Roux, C., Tani, A.: Steady solutions of the Navier-Stokes equations with threshold boundary conditions. Math. Methods Appl. Sci. 30, 595-624 (2007)

[14] Ayadi, M., Baffico, L., Gdoura, M.K., Sassi, T.: Error estimates for Stokes problem with tresca friction conditions. ESAIM Math. Model. Numer. Anal. 48, 1413-1429 (2014)

[15] Li, Y., Li, K.: Penalty finite element method for Stokes problem with nonlinear slip boundary conditions. Appl. Math. Comput. 204, 216-226 (2008)

[16] Kashiwabara, T.: On a strong solution of the non stationary Navier Stokes equations under lip or leak boundary conditions. J. Differ. Equ. 254, 756-778 (2013)

[17] Djoko, J.K.: Discontinuous Galerkin finite element discretization for steady Stokes flows with threshold slip boundary conditions. Quaest. Math. 36, 501-516 (2013)

[18] Bartels, S.: Quasi optimal estimates for implicit discrerizations of rate independent evolutions. Siam J. Numer. Anal. 52(2), 708-716 (2014)

[19] Duvaut, G., Lions, J.-L.: Inequalities in Mechanics and Physics. Grundlehren der Mathematischen Wissenschaften, vol. 219. Springer-Verlag, Berlin (1976)

[20] Glowinski, R.: Numerical Methods for Nonlinear Variational Problems. Scientific Computation, 2nd edn. SpringerVerlag, Berlin (2008)

[21] Girault, V., Raviart, P.A.: Finite Element Methods for Navier-Stokes Equations: Theory and Algorithms. SpringerVerlag, Berlin (1986) 